\title{
Is the Number of Neighbors in Ad Hoc Wireless Networks a Good Indicator of Connectivity? ${ }^{\dagger}$
}

\author{
Ozan. K. Tonguz ${ }^{1}$ and Gianluigi Ferrari ${ }^{1,2}$ \\ ${ }^{1}$ Department of Electrical and Computer Engineering, Carnegie Mellon University \\ Pittsburgh, PA 15213-3890, USA - Email: tonguz@ece.cmu.edu, gferrari@cmu.edu \\ ${ }^{2}$ Dipartimento di Ingegneria dell'Informazion, Università di Parma, Parma, I-43100, Italy
}

\begin{abstract}
Finding the minimum necessary number of neighbors required to provide full connectivity in wireless networks is an important problem which has been addressed by several researchers. In this paper, we show that the number of neighbors is a meaningful parameter to describe the connectivity only if the ad hoc wireless network has a perfectly uniform node distribution, i.e., the nodes lie approximately on the vertices of a regular grid. It is also shown that, in the case of non-uniform ad hoc wireless networks the number of neighbors becomes less meaningful. In this case, we propose instead, as indicator of the connectivity level, a reasonable measure of the likelihood of broadcast percolation for a given average number of neighbors.
\end{abstract}

\section{INTRODUCTION}

Ad hoc wireless networks have recently become very popular, due to the ever increasing demand for ubiquitous connectivity without fixed infrastructures. The nodes of such networks need to be connected in order to guarantee the possibility for a source node to reach, through multiple hops, any other node in the network.

Connectivity is often associated with the number of neighbors of a node. However, definitions of connectivity and neighbor may be different, and each of them may lead to a different conclusion. In other words, is it possible to always identify the minimum number of neighbors needed for connectivity? In this paper, we attempt to show that the number of neighbors is an optimal indicator of the connectivity level only for wireless networks with perfectly uniform node distribution. In particular, in this study the following assumptions are made.

- Circuit switching with multiple disjoint routes is used.

- A node generates information only after reserving a route. In other words, no buffering is considered. As an example, this scenario models the case of ad hoc wireless networks for real-time voice applications.

- We assume an ideal scenario where there is no internode interference (INI). This ideal assumption allows to evaluate the impact of the multi-hop nature of the wireless transmission. We also comment on what happens in a more realistic scenario with INI.

- Shortest path routing is also assumed at the moment a route is created (the route creation phase is not considered, since it is beyond the scope of this paper).

†This research was funded in part by Army Research Offi ce (ARO) under Contract No. DAAD19-02-1-0389. Any opinions, fi ndings, and conclusion or recommendations expressed in this publication are those of the authors and do not necessarily reflect the views of the Army Research Office.
Based on a maximum acceptable bit error rate (BER) constraint at the end of a multi-hop communication path, we propose a suitable definition of transmission range. According to this definition, it is possible to show that the minimum number of neighbors for full connectivity in ad hoc wireless networks with perfectly uniform node distribution is around $\pi$.

Unlike a scenario characterized by a perfectly uniform network topology, we then consider a network where the nodes are randomly distributed inside the network area. In this case, by describing the node distribution as a two-dimensional Poisson process, we derive an intuitive measure of the likelihood of broadcast percolation as a function of the number of neighbors. We also show that for networks with a very large number of nodes, the required number of neighbors for full connectivity is $O(\ln N)$, in agreement with the results in [1].

\section{Perfectly Uniform Node Distribution}

In this section we investigate the value of the minimum number of neighbors necessary to guarantee full connectivity in perfectly uniform ad hoc wireless networks. In particular, based on the communication-theoretic approach introduced in [2]-[4], we first show that the number of neighbors should be around $\pi$, i.e., between 3 and 4 . In order to understand in more depth the meaning of this result, we then consider what happens in a perfectly uniform and connected ad hoc wireless network where each node has 2 neighbors.

\section{A. A Communication-Theoretic Approach}

The derivation of an average uniform model requires the introduction of some geometric regularity. We assume that $N$ nodes are placed inside a planar surface of area $A$ and are uniformly distributed. We define by $\rho_{S} \triangleq \frac{N}{A}$ the node spatial density. We assume that the average distance between any pair of neighboring nodes is $r_{L}$. The concept of Voronoi tessellation [5] can be used to give a more precise definition of uniform node distribution. In [6], the authors prove a lemma according to which for every $\epsilon>0$, there is a Voronoi tessellation of the plane with the property that every Voronoi cell ${ }^{1}$ contains a disk of radius $\epsilon$ and is contained in a disk of radius $2 \epsilon$. Based on this characterization of Voronoi

${ }^{1}$ Given a distribution of nodes, we recall that the Voronoi cell of a node is defi ned as the set of all points, in the plane, which are closer to that node than to any of the other nodes. 
tessellation, the following definition of uniform distribution will be used in the remainder of this paper.

Definition 1: A node distribution is considered uniform with average neighboring distance $r_{L}$ if there exists a Voronoi tessellation such that every Voronoi cell is contained in a disk of radius $r_{L}$.

In other words, a uniform node distribution is such that the local structure is almost the same everywhere-for instance, this is not true in the case of a random distribution, where there could be significant variations between different regions of the network. Various uniform geometric distributions are possible. We indicate by $N_{0}$ the average number of neighbors (i.e., at average distance) of any node. It is possible to show that, in the case with an average uniform node spatial distribution, the neighbors of each node are at a distance $r_{L} \approx 1 / \sqrt{\rho_{S}}$ from it.

Indicating by $\mathrm{BER}_{L}$ the BER at the end of a single link, assuming that (i) there is regeneration (i.e., detection and possibly error correction) at each intermediate node, and that (ii) the uncorrected errors made in successive links accumulate, it is possible to show that the BER at the end of the $n$ th link of a multi-hop route, indicated by $\mathrm{BER}^{(n)}$, can be expressed as $\mathrm{BER}^{(n)} \approx 1-\left(1-\mathrm{BER}_{L}\right)^{n}$. The link BER is a decreasing function of the signal-to-noise ratio (SNR) at the ending node of the link (indicated by $S \mathrm{SR}_{L}$ ), the modulation, possible channel coding, and channel characteristics.

Imposing a constraint in terms of the maximum BER, indicated as $B E R^{\max }$, over an average multi-hop route, it is possible to show that there exists a minimum link SNR, indicated as $S_{N R} R_{L}^{m i n}$, required to fulfill the BER constraint. The transmission range can be defined as the distance at which the SNR corresponds to the minimum value $S_{N R}{ }_{L}^{m i n}$. A neighbor of a given node is then at a distance not larger than the transmission range. Assuming that the radio transmission pattern of each node is omnidirectional, it follows that the number of neighbors of a node can be written as $N_{0}=$ $\rho_{S} \pi r_{T}^{2}$. If there is no INI, then it is always possible, by sufficiently increasing the transmitted power, to guarantee that the SNR at the end of a minimum length hop is above any considered threshold $\mathrm{SNR}_{L}^{\mathrm{min}}$. This assumption can also be given a complementary interpretation. If the transmitted power is fixed, for any minimum required $S N R$ value $S_{N R}^{\min }$ at the end of a communication link, there exists a critical node spatial density $\rho_{S}^{\min }$ such that if $\rho_{S} \geq \rho_{S}^{\min }$ then the SNR at the end of a communication link is larger than $\mathrm{SNR}_{L}^{\mathrm{min}}$. The following proposition can be proved.

Proposition 1: For a given maximum tolerable BER at the end of any possible multi-hop communication path in a uniform ad hoc wireless network with finite area, the minimum number of neighbors required for full connectivity is, on average, $\pi$. In other words, indicating by $N_{0}$ the number of neighbors of a node,

$$
\begin{aligned}
\forall\left(\mathrm{BER}^{\max }, n_{\max }\right), \quad \exists P_{t}^{\min }=P_{t}^{\min }\left(\mathrm{BER}^{\max }, n_{\max }\right): \\
P_{t}=P_{t}^{\min } \Rightarrow N_{0}=\pi, \mathrm{BER}^{\left(n_{\max }\right)}=\mathrm{BER}^{\max } .
\end{aligned}
$$

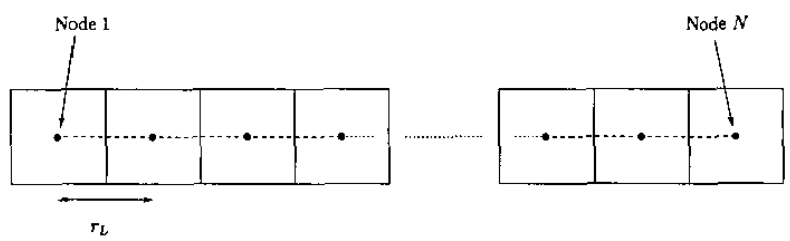

Fig. 1. Ad hoc wireless network where the nodes are placed over a line.

Proposition 1 can be given a simple and intuitive interpretation. Given a uniform network topology with fixed node spatial density, in order to support multiple hops it is necessary that each node reaches its nearest neighbors with an amount of power which guarantees sufficient regeneration of the transmitted signal along a multi-hop route in order to have a minimum prescribed BER at the final node. This situation can happen if the transmitted power is larger than a minimum critical value (for fixed node spatial density) or if the node spatial density is larger than a minimum critical value (for fixed transmitted power). At this point, full connectivity, through multi-hop routing, is guaranteed and the minimum required number of neighbors is $\pi$.

\section{B. What Happens with $N_{0}=2$ Neighbors?}

At this point, one might argue that there can exist a network which guarantees full connectivity with a number of neighbors lower than $\pi$. This could be the case, for example, of a wireless network where the nodes lie on a line, as indicated in Fig. 1. In this case, the network area can be considered as the union of the squares (of side $r_{L}$ ) centered at the nodes. Let us assume that there are $N$ nodes, as in Section II-A. We propose a simple derivation of the average number of hops. In particular, we compare the result in the case of a circular network area with grid node distribution with that in the case of a network where the nodes lie on a line.

- As shown in Subsection II-A, the maximum number of hops is given by $n_{\max }=d_{A} / r_{L} \approx 2 \sqrt{N / \pi}$. Assuming that the number of hops is uniformly distributed between 1 and $n_{\max }$, it follows that the average number of hops is (for sufficiently large $N$ ) $\bar{n}_{h} \approx \sqrt{N / \pi}$.

- In the case of a linear network, the maximum number of hops is $n_{\max }=N$. Assuming in this case as well that the number of hops is a random variable uniformly distributed between 1 and $N$, it follows that the average number of hops is (for sufficiently large $N$ ) $\bar{n}_{h} \approx N / 2$.

We evaluate the BER at the end of an average communication route. For simplicity, we consider the case of transmission, affected by free space loss, over an additive white Gaussian noise channel. We assume that the thermal noise power can be written as $F k T_{0} B$, where $F$ is the noise figure, $k=$ $1.38 \times 10^{-23} \mathrm{~J} / \mathrm{K}$ is the Boltzman's constant, $T_{0}$ is the room temperature, and $B$ is the transmission bandwidth. Assuming that the power transmitted by a node is $P_{t}$, the received power at distance $d$ from the transmitting node, indicated by $P_{r}^{(d)}$, can be written, according to the Friis free space formula, as

$$
P_{r}^{(d)}=\frac{\alpha P_{t}}{d^{2}}=\frac{G_{t} G_{r} \lambda_{c}^{2} P_{t}}{(4 \pi)^{2} f_{l} d^{2}}
$$




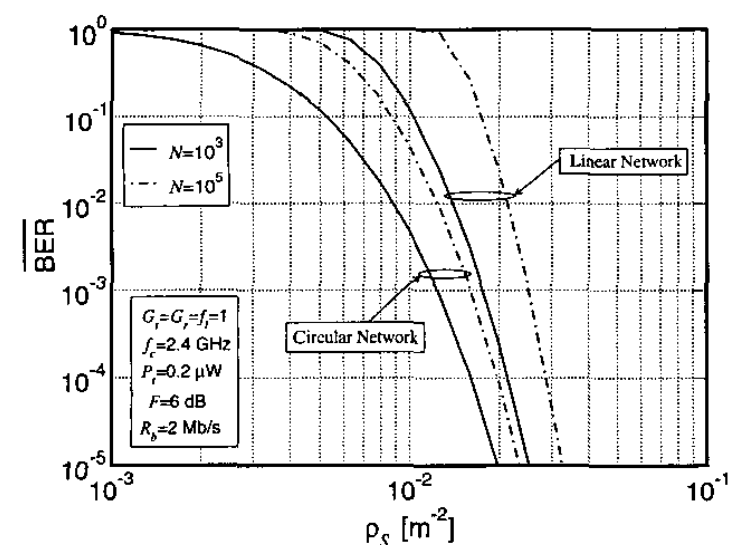

Fig. 2. BER performance in the case with a circular network area and a linear network area.

where: $G_{t}$ and $G_{r}$ are the transmitter and receiver antenna gains, respectively; $\lambda_{c}=c / f_{c}$ is the wavelength corresponding to the carrier frequency $f_{c}$ ( $c$ is the speed of light); $f_{l} \geq 1$ is a loss factor which takes into account the losses not related to propagation. We consider uncoded binary phase shift keying (BPSK) as the modulation format. A performance comparison in terms of average $\mathrm{BER}$, i.e., $\overline{\mathrm{BER}}=\mathrm{BER}^{\left(\bar{n}_{h}\right)}$, versus node spatial density, in the cases with a circular and a linear area network, is shown in Fig. 2. In particular, the transmitted power is fixed, and two possible values of the number of nodes are considered. As one can see, the average BER performance in a linear network is worse than in the case with a circular area network. This formalizes the intuitive observation that an ad hoc wireless network where each node has 2 neighbors is "less connected" than an ad hoc wireless network where each node has, on average, $\pi$ neighbors: in other words, a higher node spatial density is needed to support the same average BER.

\section{What Happens if There is Inter-Node Interference?}

From other results in [4], it seems reasonable to assume that the INI power in the case of circuit-switched multi-hop ad hoc wireless networks can be considered proportional to the transmitted power $P_{t}$ and to the node spatial density $\rho_{S}$. In other words, $P_{I N T} \propto \rho_{S} P_{t}$. Extending the approach proposed in Subsection II-A, it is possible to prove the following theorem.

Proposition 2: In a uniform ad hoc wireless network communication scenario with INI where $P_{I N T} \propto \rho_{S} P_{t}$, there might be two mutually exclusive situations.

- There is not full connectivity, regardless of the transmitted power. In other words,

$$
\begin{aligned}
& \exists\left(\mathrm{BER}^{\max }, n_{\max }\right) \quad: \forall P_{t}, N_{0}, \\
& \mathrm{SNR}_{L}<\mathrm{SNR}_{L}^{\min }=\mathrm{SNR}_{L}^{\min }\left(\mathrm{BER}^{\max }, n_{\max }\right) .
\end{aligned}
$$

- There is full connectivity, on average, and the minimum required number of neighbors is $\pi$. In other words,

$$
\begin{gathered}
\forall\left(\mathrm{BER}^{\mathrm{max}}, n_{\max }\right), \quad \exists P_{t}^{\min }=P_{t}^{\min }\left(\mathrm{BER}^{\max }, n_{\max }\right): \\
P_{t}=P_{t}^{\min } \Rightarrow N_{0}=\pi, \mathrm{BER}^{\left(n_{\max }\right)}=\mathrm{BER}^{\max } . \text { (4) }
\end{gathered}
$$

In other words, in a realistic network scenario with INI there might be situations where increasing the transmitted power in order to make the network connected could be a waste of resources.

\section{NON-UNIfORM NODE DISTRIBUTION}

The case of networks where the node distribution is random has long been studied in the context of various theories. In the following, we first review the existing literature, and we propose a simple approach for the evaluation of the likelihood of broadcast percolation in non-uniform ad hoc wireless networks.

\section{A. Existing Literature}

It is possible to group the approaches that appeared in the literature into the following main categories.

- In [7], the optimal transmission radius $r_{T}^{o p t}$ is found considering, as optimality criterion, the network throughput, suitably reformulated in terms of one-hop progress. In particular, the considered wireless communication networks are affected by INI and slotted Aloha MAC protocol is considered. It is shown that the minimum number of neighbors corresponds to a "magic number" equal to 6.

- Another possible approach is based on the theory of continuum percolation [8]. This approach is considered, in the context of random plane networks, in [9], where the minimum number of neighbors needed for full connectivity in an average uniform network with finite area is estimated, through simulations, as 3.2 (which is very close to our result, i.e., $\pi$ ). In [10], the authors show that, in the case of a network with finite area, the number of neighbors should be between 2.2 and 10.5 . Broadcast percolation is considered in [11], where exact results are given in the one-dimensional case, and numerical results are presented in a two-dimensional percolation scenario.

- A final approach is related to the analysis of ad hoc wireless networks in the context of random graph theory [12]. The minimum number of neighbors needed for connectivity is evaluated in [13]. This issue is further explored and refined in [1], where it is proved that a wireless network is asymptotically connected, for large number of nodes, if the number of neighbors of each node is $O(\ln N)$.

\section{B. A Simple Approach to the Evaluation of the Likelihood of Broadcast Percolation}

In general, a well connected wireless network should be such that a node, upon receiving a packet from another node in one direction, can forward the packet to all remaining three possible directions, as indicated in Figure 3 (a). If the number of neighbors of the central node (within a circle of radius corresponding to the transmission range $r_{T}$ ) is lower than four, then the packet can not be broadcast ahead in the remaining directions, as indicated in Figure 3 (b), (c), and (d). Intuitively, this means that the broadcast will not percolate uniformly in 


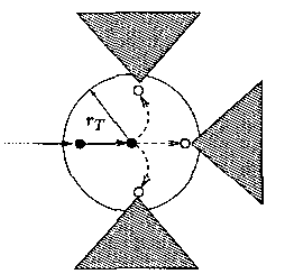

(a)

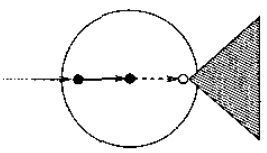

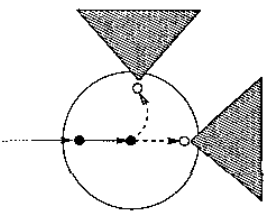

(b)

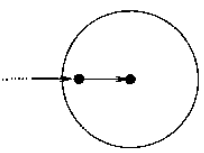

Fig. 3. Possible broadcast situations: (a) full broadcast, (b) partial broadcast, (c) limited broadcast (d) no broadcast.

the network. We indicate this event as non-local percolation (NLP) and its corresponding probability as $p_{N L P}$. The number of neighbors of a node, i.e., within transmission range $r_{T}$, is $N_{0}=\rho_{S} \pi r_{T}^{2}$. Recalling the memoryless property of a Poisson distribution, the probability of encountering a NLP situation can be written as

$$
p_{N L P}=\sum_{i=0}^{2} P\left\{i \mid \pi r_{T}^{2}\right\}=e^{-N_{0}}\left(1+N_{0}+\frac{N_{0}^{2}}{2}\right) .
$$

Assuming that $N$ nodes are approximately distributed in an area $A=N / \rho_{S}$ and that the area $A$ is circular, we still assume (as in the case with uniform grid topology) that the average number of hops of a communication route in a circular area is $\sqrt{N / \pi}$. We can roughly estimate the probability of having at least one NLP situation over an average communication route $^{2}$, indicated as $p_{N L P}^{(N)}$, in the following way:

$$
p_{N L P}^{(N)}=1-\left(1-p_{N L P}\right)^{\sqrt{\frac{N}{\pi}}}
$$

The probability $p_{N L P}^{(N)}$ can be considered as an indicator of the likelihood of a broadcast to percolate rapidly across the network (the lower is $p_{N L P}^{(N)}$, the faster is the percolation), and this can also be interpreted as a degree of connectedness of the network. Note that $p_{N L P}^{(N)}$ can not be interpreted as an exact indicator of the connectivity in the network. In fact, even if an NLP event happens at a given hop in a communication route, the packet could still reach its destination by following an alternative (longer) route. Intuitively, one can assume that if $p_{N L P}^{(N)} \leq \delta$, for values of $\delta$ sufficiently low $(\delta \ll 1)$, connectivity is guaranteed almost surely. For large values of $N$, through a first-order Taylor series expansion, from (5) and (6) it is possible to show that the condition $p_{N L P}^{(N)} \leq \delta$ can be equivalently rewritten as follows:

$$
N_{0} \geq \frac{1}{2} \ln N
$$

\footnotetext{
${ }^{2}$ The calculation of the average number of hops is based on the assumption of uniform distribution. In the case of a Poisson node distribution this might not be the case. However, the results are qualitatively the same, since we can assume that the average number of hops in a communication route is $O(\sqrt{N})$ in any case.
}

The result in (7) agrees with the result obtained in [1]. In particular, this asymptotic result holds regardless of the definition (in terms of the number of terms of the Poisson distribution summed) of $p_{N L P}^{(N)}$ (as a function of $p_{N L P}$ ) and for any considered critical maximum value $\delta$.

\section{CONCLUDING REMARKS}

Numerous approaches have appeared in the literature for the evaluation of the number of neighbors as an indicator of the connectivity in wireless networks. The "optimal" number of neighbors depends on the considered criterion and on the network characteristics. Two fundamental categories of network topologies can be identified: (i) a scenario where the node distribution is perfectly uniform and (ii) a scenario where the node distribution is random. In the case of ad hoc wireless network with perfectly uniform node distribution, it is shown that the number of neighbors is a good indicator of the connectivity level of the network and that the minimum number is around $\pi$. In the case of non-uniform networks, the number of neighbors becomes less meaningful. Although this scenario has been widely considered in the literature, we proposed an alternative simple approach for the evaluation of the likelihood of broadcast percolation. For very large values of the number $N$ of nodes in the network, our approach suggests that the number of neighbors $N_{0}$ should be on the order of $\ln N$, i.e., $O(\ln N)$, in agreement with the predictions of [1].

\section{REFERENCES}

[1] F. Xue and P. R. Kumar, "The number of neighbors needed for connectivity of wireless networks," Wireless Networks, April 2003.

[2] O. K. Tonguz and G. Ferrari, "A Communication-Theoretic Framework for Ad Hoc Wireless Networks," Carnegie Mellon University, ECE Dept., Tech. Rep., February 2003.

[3] G. Ferrari and O. K. Tonguz, 'Performance of circuit-switched ad hoc wireless networks with Aloha and PR-CSMA MAC protocols," in Proc. IEEE Global Telecommun. Conf. (GLOBECOM), San Francisco, USA, December 2003.

[4] — 'Mac protocols and transport capacity in ad hoc wireless networks: Aloha versus PR-CSMA," in Proc. IEEE Military Comm. Conf. (MILCOM), Boston, USA, October 2003.

[5] A. Okabe, B. Boots, and K. Sugihara, Spatial Tesselations Concepts and Applications of Voronoi diagrams. New York, USA: John Wiley \& Sons, 1992.

[6] P. Gupta and P. R. Kumar, "The capacity of wireless networks," IEEE Trans. Inform. Theory, vol. 46, pp. 388-404, March 2000.

[7] L. Kleinrock and J. A. Silvester, 'Optimum transmission radii for packet radio networks or why six is a magic number," in Nat. Telecommun. Conf., dec 1978, pp. 4.3.1-4.3.5.

[8] R. Meester and R. Roy, Continuum Percolation. Cambridge, U.K. Cambridge University Press, 1996.

[9] E. N. Gilbert, 'Random plane networks," SIAM J., vol. 9, no. 4, pp. 533-543, December 1961.

[10] T. K. Philips, S. S. Panwar, and A. N. Tantawi, 'Connectivity properties of a packet radio network model," IEEE Trans. Inform. Theory, vol. 35 , no. 5, pp. 1044-2047, September 1989.

[11] Y.-C. Cheng and T. G. Robertazzi, 'Critical connectivity phenomena in multihop radjo models," IEEE Trans. Commun., vol. 37, no. 7, pp. 770-777, July 1989.

[12] P. Erdös and A. Rényi, "On the evolution of random graphs," Proceed. ings of Hungarian Academy of Sciences, pp. 17-61, May 1960.

[13] C. Bettstetter, 'On the minimum node degree and connectivity of a wireless multihop network," in Proc. ACM Int. Symp. on Mobile Ad Hoc Network. and Comput. (MOBIHOC), Lausanne, Switzerland, June 2002, pp. $80-91$. 